\title{
Primary ovarian carcinoid tumor arising within a mature cystic teratoma in a 32-years-old patient.
}

\author{
Manuel Dominguez Gonzalez*, Sofia Vazquez Navarrete, Raquel Jimenez Pena, Maria Carmen Escamez Leon \\ Department of Gynecology and Obstetrics, Hospital La Linea, Avenida Menéndez Pelayo, 103 s/n, La Linea de la \\ Concepción, Cadiz, Spain
}

\begin{abstract}
Mature cystic teratomas (MCT) or dermoid cyst represent 10-20\% of all ovarian neoplasm. Primary ovarian carcinoids are rare, accounting for $0,3 \%$ of all carcinoid tumors. We report a case of a 32-years-old woman diagnosed with primary carcinoid tumor of the left ovary arising in MCT.

Vaginal ultrasonography showed left cystic adnexal image of $60 \times 40 \mathrm{~mm}$, with fine echogenic dotting and a more refringent area in caudal pole. Doppler negative. Negative tumor markers.

Pelvic MRI: Pelvic tumor of approximately $50 \times 61 \mathrm{~mm}$ of suprauterine location with a thin hypointense capsule with well-defined borders and fat-like signal intensity, which impresses of mature cystic teratoma as the first diagnostic possibility.Laparoscopy and left anexectomy was performed. The histopathological study reports, mature cystic teratoma of $5 \mathrm{~cm}$ with a single focus $(0.6 \mathrm{~cm})$ of carcinoid tumor.

It is difficult to make a diagnosis of primary ovarian carcinoid tumor arising within a mature cystic teratoma of the ovary preoperatively. A thorough histopathological study of the tumor is very important and the surgical treatment adapted according to the characteristics of the patient.
\end{abstract}

Keywords: Carcinoid tumor, Dermoid cyst, Ovary, Teratoma.

\section{Introduction}

Mature cystic teratomas (MCT) or dermoid cyst represent 10$20 \%$ of all ovarian neoplasm [1]. Primary ovarian carcinoids are rare, accounting for $0.3 \%$ of all carcinoid tumors and the majority of them are associated with MCT [2].

Malignant transformation of teratoma (TMT) is uncommon condition occurring in 1-3\% of all mature cystic teratomas [3] and is very rare, with a very small number of published cases. We report a case of a 32-year-old woman diagnosed with primary carcinoid tumor of the left ovary arising in MCT.

\section{Case Report}

A 32-year-old patient with a casual diagnosis of left adnexal mass in a routine exam.

Asymptomatic personal history: No allergies.

Congenital cataracts: Raynaud's disease.

Gynecological and obstetrical history: Menarche at age 12 years. Regular menstrual cycles. Two deliveries and one abortion.

Contraceptive method: Copper diu. Negative PAP's smears.

Exploration without pathological findings: Uterus of normal size, mobile cervix. It is not possible to feel mass in bimanual touch. Vaginal ultrasonography demonstrated uterus of normal size and morphology. Diu normally inserted. Left cystic adnexal image of $60 \times 40 \mathrm{~mm}$, with fine echogenic dotting and a more refringent area in caudal pole. Doppler negative, douglas free, right ovary is not seen. It is not possible to clearly visualize the mass through abdominal ultrasound. Negative tumor markers: CA125: $10.2 \mathrm{u} / \mathrm{ml}$. CA19.9: $16.5 \mathrm{u} / \mathrm{ml}$. Negative for pregnancy test.

Pelvic magnetic resonance imaging (MRI): Pelvic tumor of approximately $50 \times 61 \mathrm{~mm}$ of suprauterine location with a thin hypointense capsule with well-defined borders and fat-like signal intensity, which impresses of mature cystic teratoma as the first diagnostic possibility. Diu normally inserted. Normal right ovary. No other findings of interest (Figure 1).

Laparoscopy and left anexectomy was performed. Examination of the abdominal cavity is normal. The left ovarian tumor is compatible with teratoma. Right ovary, fallopian tubes and uterus are normal. No lymphadenopathy or other pelvic lesions are present. The sample is sent to the Patholoy Department. The intervention went without incident. Postoperative evolution was favorable (Figure 2).

Macroscopically the surgical specimen was the left adnexa with fallopian tube $5 \mathrm{~cm}$ lenght and enlarged ovary, $5.5 \times 3.5 \mathrm{~cm}$. When slicing the specimen it was cystic with a small $(1 \mathrm{~cm})$ solid area. The cyst was filled with sebaceous material and hair. Histologically the tumor was integrated by poligonal cells with light and eosinophilic, cytoplasm with rounded nuclei with mild pleomorphism. No mitotic figures were identified. Those cells exhibited a trabecular and insular pattern (Figure 3). In other areas the tumor had the elements of a typical cystic teratoma, with structures resembling skin appendages, an queratin. The diagosis 
Citation: Gonzalez MD, Navarrete SV, Pena RJ, et al. Primary ovarian carcinoid tumor arising within a mature cystic teratoma in a 32-years-old patient. Gynecol Reproduct Endocrinol -UK. 2018;2(1):2-4

was: Mature cystic teratoma with a carcinoid $(0.6 \mathrm{~cm})$ with combined pattern insular and trabecular organ-confined, without capsular or vascular invasion. Immunohistochemistry was performed and the tumor was positive for CD56, chromogranin (Figure 4), synaptophysin and focally CK7. The Ki-67 index was $10 \%$ (eyeballing). After several months of follow-up, the patient continues asymptomatic and neimaging tests.

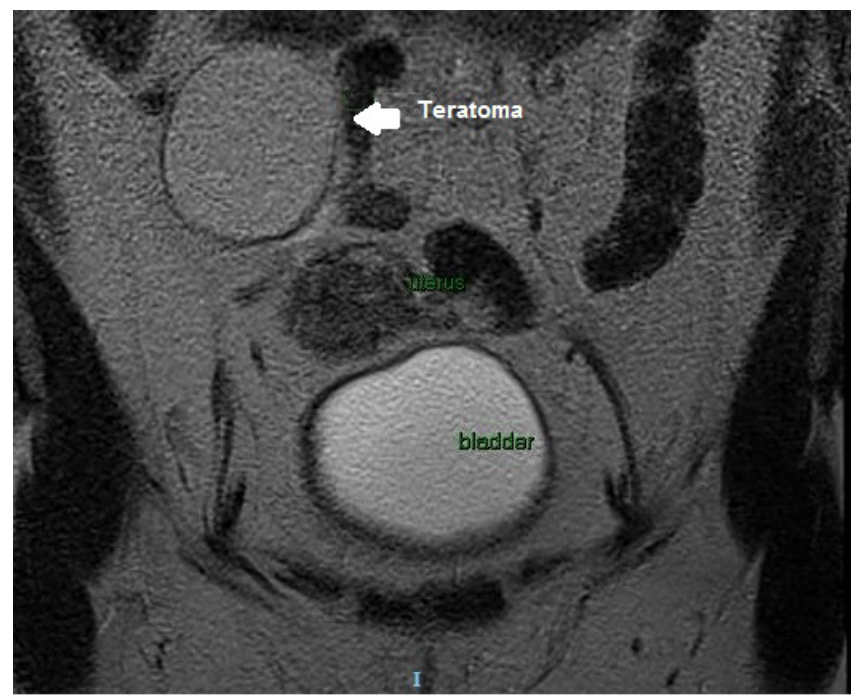

Figure 1: Pelvic MRI, coronal view.

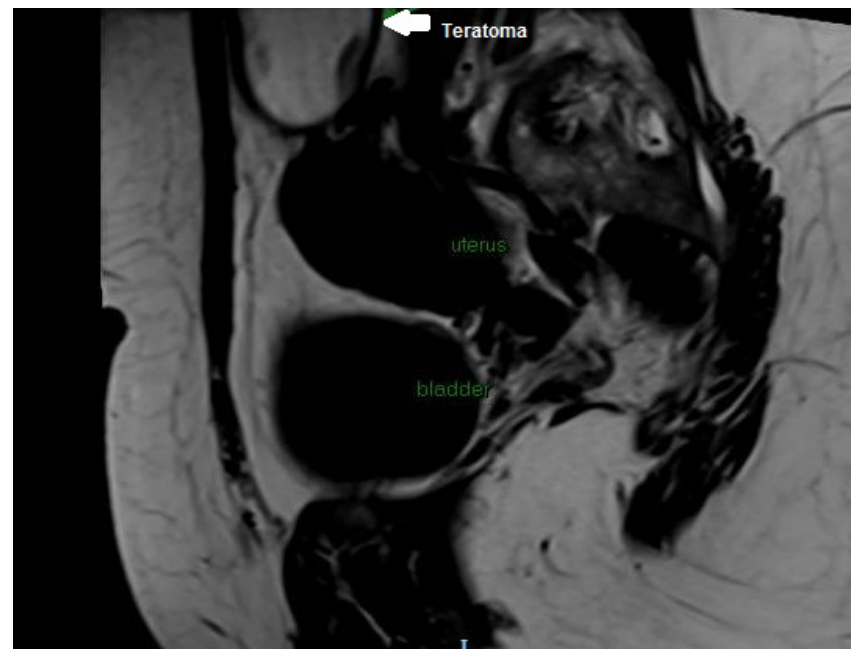

Figure 2: Pelvic MRI, sagittal view.

\section{Discussion}

It is an infrequent pathology, but it must be taken into account, since there is a risk of not removing the carcinoid tumor completely. Although in most cases the evolution is described as favorable, we can not guarantee that it will always be the case, since there are few cases described in the literature.

Teratomas consist of diverse tissues derived from the three germ cell layers, the ectoderm, mesoderm, and endoderm. Carcinoid tumors in ovarian and testicular teratomas are considered to be derived from neuroendocrine cells of the gastrointestinal or respiratory epithelium [4]. Carcinoid tumors most commonly occur in the gastrointestinal tract.

Carcinoid tumor can be seen in the ovary as a metastasis of a primary tumor located in the gastrointestinal tract or elsewhere, as a component of mature cystic teratoma or as a primary pure neoplasm of this organ [2]. The large majority of primary ovarian carcinoids are unilateral, but in $16 \%$ of cases the contralateral ovary is involved by a cystic teratoma or a mucinous neoplasm [5].

The established criteria used for differentiating metastatic tumors of the ovary from primary ovarian tumors are: tumor bilaterality, presence of multiple ovarian nodules and the finding of a primary tumor in the gastrointestinal tract, all favor metastasis to the ovary [6].

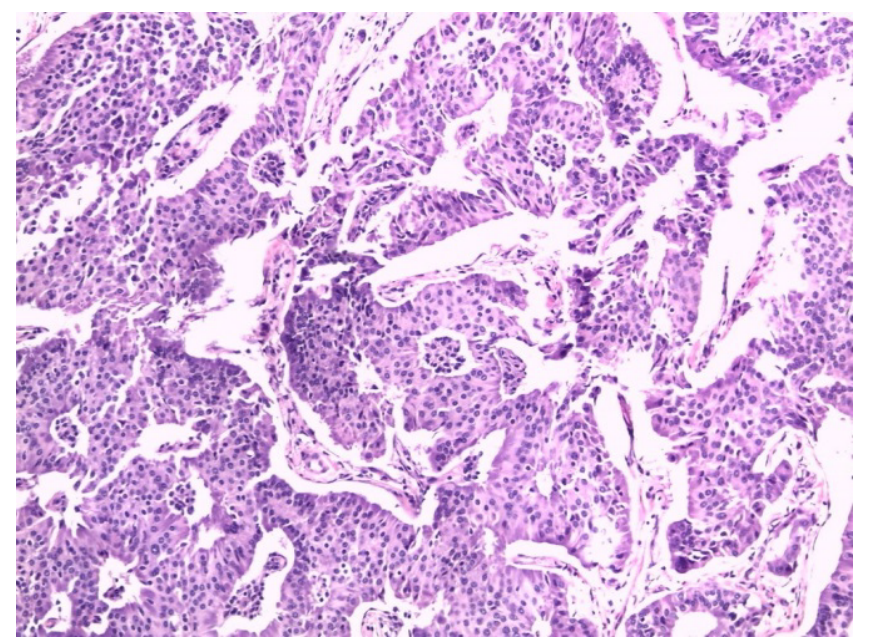

Figure 3: Trabecular and insular pattern.

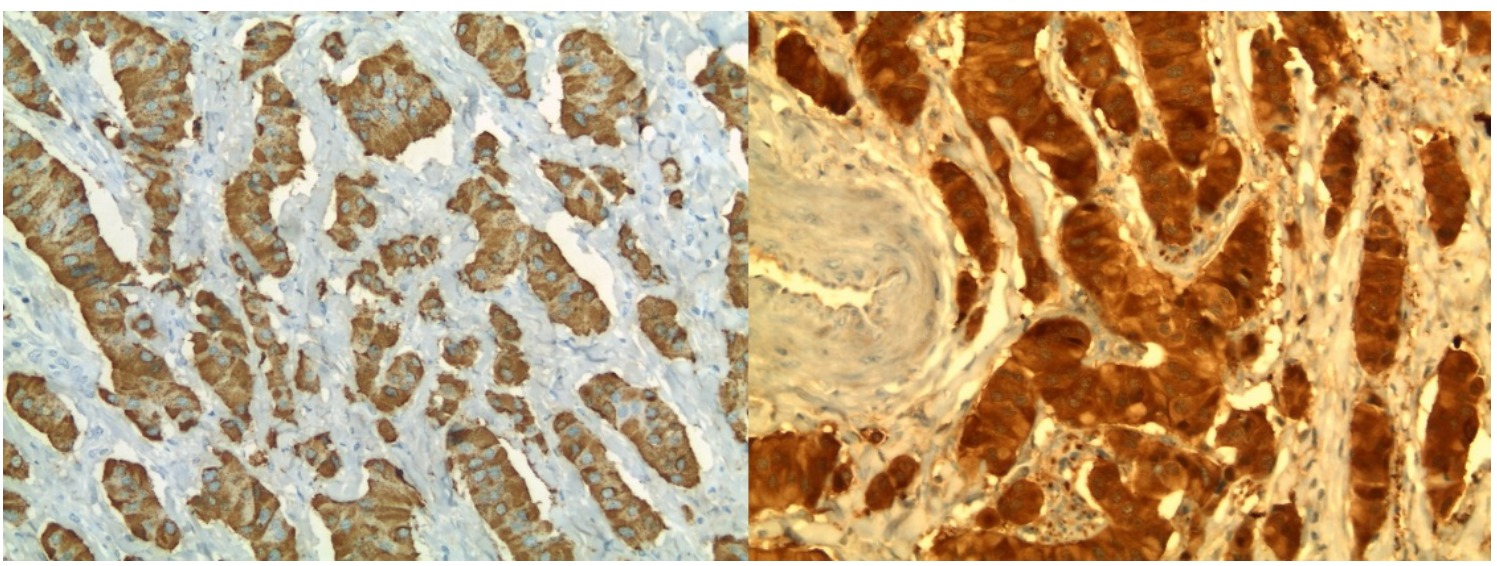

Figure 4: Immunohistochemistry was performed and the tumor was positive for CD56, chromogranin, synaptophysin and focally CK7. 
Our patient has a primary carcinoid tumor because it is a single tumor in the left ovary, with an intact capsule, without vascular invasion, or other suspicious lesions in the abdominal cavity.

Primary ovarian carcinoid tumors are classified into four types, i.e, the insular, trabecular, mucinous, struma carcinoid and mixed types. The most common type is the insular type [7]. Primary ovarian carcinoid tumors, in particular one third of the insular type, have been associated with the carcinoid syndrome (classical triad of flushing of upper extremities and face, wheezing and diarrhea).

In most case, a definitive diagnosis is possible only on postoperative examination of multiple tissue sections. It is possible to suspect the presence of a carcinoid tumor in ovarian teratoma preoperatively if the patient presents symptoms of carcinoid syndrome:

Skin flushing: The skin of the face and upper chest feels hot and changes color, ranging from pink to purple. Episodes of redness can last from a few minutes to a few hours or more.

Facial skin lesions: Purplish areas of spiderlike veins may appear on the nose and upper lip.

Diarrhea: In people with carcinoid syndrome, frequent watery stools accompanied by colic may occur.

Difficulty breathing: Asthma-like signs and symptoms, such as wheezing and shortness of breath, may occur at the same time you experience skin flushing.

Palpitations: The tumor has been detected incidentally in our case and classified as mixed (insular and trabecular). The patient did not present with carcinoid syndrome. The immunohistochemistry was performed to confirm the diagnosis given that these markers (CD56, chromogranin, synapthophysin) have previously been reported in ovarian neuroendocrine tumors [8-10].

It is difficult to establish an optimal surgical treatment because it is such a rare disease. An attempt is made to adapt the surgical aggressiveness according to the age of the patient. Conservative surgery (anexectomy) is reserved for young women, especially nulliparous or who wish to preserve fertility. Hysterectomy and bilateral salpingo-oophorectomy are advised in postmenopausal patients.

\section{Conclusions}

- Primary carcinoid tumors arising in mature teratoma are tumors with low malignancy potential in most cases [4-11].

- It is a rare pathology of which there are not many cases published in the literature.

- The prognosis and clinical behavior of these tumors have not yet been clarified [12,13].

- It is difficult to make a diagnosis of TMT of the ovary preoperatively. If the patient presents carcinoid syndrome we should suspect a carcinoid tumor associated with teratoma.

- A thorough histopathological study of the tumor is mandatory and the surgical treatment adapted according to the characteristics of the patient.
- It would be necessary to expand knowledge of this pathology, making a systematic review of all cases published in the literature.

\section{References}

1. Robert JK, Maria LC, Simon H, et al. WHO Classification of tumors of female reproductive organs. $4^{\text {th }}$ edn. Lyon: International Agency for Research on Cancer (IARC) 2013;60-66.

2. Sharma R, Biswas B, Wahal SP, et al. Primary ovarian carcinoid in mature cystic teratoma: A rare entity. Clin Cancer Investig J 2014;3:80-83

3. Kim SM, Choi HS, Byun JS, et al. Mucinous adenocarcinoma and stromal carcinoid tumor arising in one mature cystic teratoma of the ovary with synchronous cervical cancer. J Obstet Gynaecol Res 2003;29:28-32.

4. Yamasaki T, Yagihashi Y, Shirahase T, et al. Primary carcinoid tumor arising in a retroperitoneal mature teratoma in an adul.: Int J Urol 2004;11:912-915.

5. Robboy SJ, Scully RE, Norris HJ et al. Carcinoid metastatic to the ovary. A clinic-pathologic analysis of 35 cases. Cancer 1974;33:798-811.

6. Huang Y, Kumarapeli A, Chen F, et al. Primary mucinous carcinoid of the ovary arising in a mature cystic teratoma: A case report with review of the literature. N A J Med Sci 2012;5:239-242.

7. Taleraman A, Kurman RJ. Blaustein's pathology of the female genital tract. 5th ed. New York: Springer. 2010.

8. Chun YK. Neuroendocrine tumors of the female reproductive tract: A literature review. J Pathol Transl Med 2015;49:450-451.

9. Niu D, LiZ, Sun L, et al. Carcinoid arising from the teratomatous bronchial mucosa in a mature cystic teratoma of the ovary: A case report. Int J Gynecol Pathol 2017.

10. Kusku E, Eroglu D, Ozdemir BH et al. Primary carcinoid tumor of the ovary: A case report. Eur J Gynaecol Oncol 2003;24:574-576.

11. Kim NR, Ha SY, Shin JW, et al. Primary ovarian mixed strumal and mucinous carcinoid arising in an ovarian mature cystic teratoma. J Obstet Gynaecol Res 2016;42:211-216.

12. Isobe $H$, Takashima $H$, Higashi $N$, et al. Primary carcinoid tumor in a horseshoe kidney. Int J Urol 2000;7:184-188.

13. Guney N, Sayilgan T, Derin D et al. Primary carcinoid tumor arising in a mature teratoma of the ovary: A case report. Eur J Gynaecol Oncol 2009;30:223-225.

\section{*Correspondence to:}

Manuel Dominguez Gonzalez

Department of Gynecology and obstetrics

Hospital La Linea

Cadiz, Spain

Tel: +34956026500

E-mail: manueldominguezg@hotmail.com 